\section{Digital Movies for Others to See}

\author{
Jerry Sedgewick \\ University of Minnesota \\ sedge@umn.edu
}

In a departure from traditional articles on Photoshop, this article will provide a look at how to save movies for the purpose of distribution to Macintosh, Windows and Linux operating systems.

More than once, I have been asked to produce and help with the distribution of digital videos that rival those downloadable trailers for big Hollywood productions. I kindly inform the clients that I do not have a staff large enough to tweak each individual frame of their video, and that their expectation must go down a few notches. Upon seeing their baffled look, an explanation ensues, much like what follows:

First of all, digital video must be compressed in order to run seamlessly from a computer. Only the most robust computers can keep up with the number of frames flashing by per second ( $~ 30$ frames for NTSC), and even these cannot play back for extended periods of time without the loss of some frames (what is called dropped frames in the digital editing world). This is seen as a sudden stop and start in the video itself so that the video is jerky versus smooth when playing back.

The exception to this rule occurs when a small number of frames are used, such as movies generated from a confocal stack. These are not "movies" in the traditional sense (the output from a movie or video camera); these are, instead, animations. Animations comprise a series of stills, each one filling $\sim 2$ to 6 frames. When these animations are generated by scientific programs, often the images are NOT compressed. For example, that is the case with the confocal program primarily designed to work with Bio-Rad files, Confocal Assistant.

These animations, however, may also need to be compressed when intended for distribution to assure smooth and universal playback. We are accustomed to compression for smaller file sizes using JPEG, GIF and PNG compression protocols. Each of these are lossy, which means that some image information is irrevocably thrown away, never to be retrieved. Each of these protocols is spatial as well. That means information from rows and columns of pixels in the im age itself are used to determine which parts can be eliminated.

Video compression can use a spatial protocol like JPEG to reduce the file size in one frame after another. However, that kind of compression may not be as efficient when working with movies. In the entertainment industry, much of what comprises movies are "talking heads." Because "talking heads" move against static backgrounds, it makes the most sense to compress the background since it remains the same frame after frame. That kind of compression looks forward $\mathrm{x}$ number of frames, or makes forward predictions, to determine what remains the same, and then compresses that visual information, leaving what moves relatively untouched. It's referred to as temporal compression. Many utilities use both spatial and temporal means when compressing video.

The plot now thickens because video operates along several standards, divided by Macintosh and Windows operating systems, and by companies, which have taken advantage of web video, such as REAL. Video requires not just the compression of frames for seamless playback, but it also requires that the computer's video play back program has some kind of utility for decompressing the video. In other words, the compressed video is programmed in such a way that it can't simply be spit out without a means for interpreting the code. Thus, these utilities are called CODECs (COmpression/ DECompression utilities), and the CODEC needs to exist on the computer in which the compression is done and on the computer in which the compressed video is played back.

That would all be very nice if only the CODEC was the same name as the file format extension. Not. The most popular formats, AVI (Windows) and .MOV (Macintosh) can use compression/decompression utilities from other sources. The.AVI format typically uses Cinepak (there's also Indeo, Windows for Video, etc.), and the .MOV format typically uses the Sorenson family of CODECs.

It gets simpler with other formats. In the instance of MPEG, the encoding utility IS called some version of MPEG (MPEG-1, MPEG-2, MP3, MPEG-4). The REAL format is a little more pleasant with its compression/decompression utility also called REAL, and the newer Windows format, WMV, uses a WMV Compression/Decompression utility. The out-of-date, but tried-and-true.GIF animation stacks do not need a CODEC utility.

Added to these matters is the video playback software program, often referred to as the architecture because these programs do more than simply play back (files are "managed" as well). QuickTime wants to play MOV formats, and it will do so as long as the compression/ decompression utility is somewhere on the computer (Sorenson comes with the Macintosh operating system, as well as Cinepak). If QuickTime on the Mac reads an AVI file, it will convert it into the MOV format and then play it back for you. The Windows media player on newer computers will play both AVI and WMV formats, but older computers do not play the WMV format, and MOV formats often need QuickTime for playback. PowerPoint on Windows will play AVI and putatively plays back MOV, but good luck trying. On the Macintosh, at least with the newer PowerPoint programs, both AVI and MOV formats can be played. The REAL architecture plays REAL and several other formats, including AVI and MOV. GIF animations play back with any network browser, such as Netscape, Mozilla or Internet Explorer. In the long run, it's the Macintosh that is far more forgiving and universal when it comes to video playback.

Finally, there is the video capture board (as opposed to the video display board). If a professional video capture board is used for video editing, then encoding/decoding occurs in hardware. While that makes editing go faster, it also introduces another hitch: movies made using a video capture board typically requires a computer also with that board in order to play back the movie you have created. In other words, your movie won't play back anywhere but on the machine where you have created it, if you save the video with default settings. What you may not have been told when purchasing a video capture board for the purposes of video editing is that you need to also buy a conversion program like Media Cleaner (this program comes with Adobe Premiere). Note: this does not apply when using firewire for video capture, or when using several popular video capture boards purchased through your microscope sales person: those boards aren't intended for editing.

Confused? You should be. The video kingdom is replete with too many choices; too many "new and better" ways to make movies and a lack of uniform standards. Again, none of these vagaries of video production and play back may be problematic for any one person's situation: these issues become problems when trying to find a "universal" movie format/CODEC. Given the explosion of live cell research and the increasing desire of reviewers to visit a web page in order to see "the video," a common format with a common CODEC and reliable playback is crucial.

For that, I will stick my neck out and recommend the MPEG1 format/CODEC, or a .GIF stack for playback on the web. While it's true that the format should be changed depending on the intent (whether for CD, CD-video, web streaming, etc.), the reality is that we don't have the time to keep up or to know all the various formats for intended output. 


\section{LEO's New Microscopes Answer All Your EM Needs}

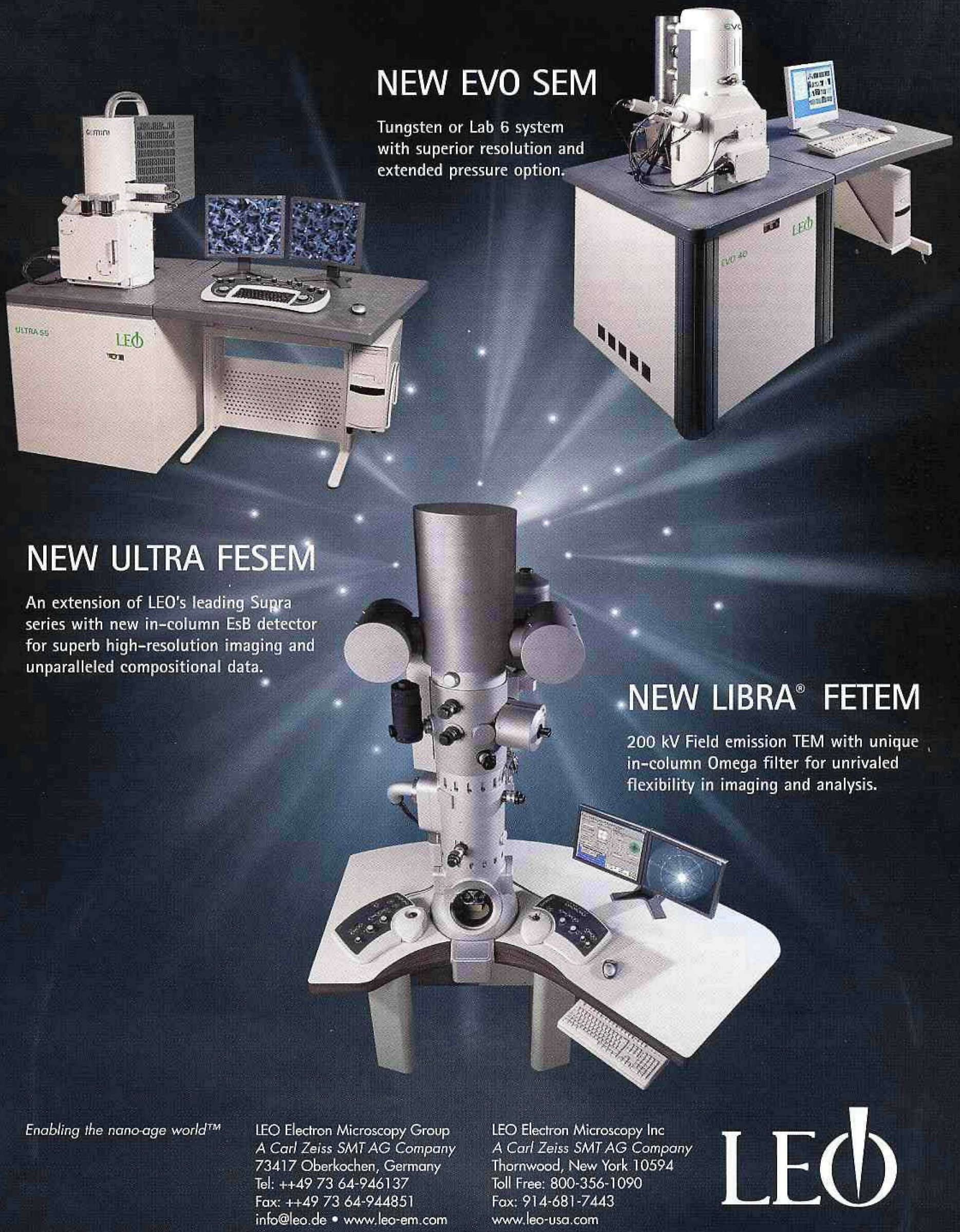


MPEG-1 can be played back in just about any program on any platform in any version of PowerPoint. The greatest advantage is that no one needs to download any particular architecture to play it back: these are on just about every computer. The intent for an MPEG-1 video is to write it to $\mathrm{CD}$ or post a link to it on the web for downloading by visitors to your page.

For viewing animations on the web site itself, GIF stacks make a nice way to easily view short animations. It's true that Flash animations work better as far as conserving on file size, but viewers need the Shockwave download in order to view the animations. Furthermore, the program is made primarily for drawings. Scientists typically want a series of images made into animation. According to the manual, this can be done in Flash, but my own experience has been that Flash only likes its image files saved in Macromedia's PNG format. Then a Flash movie can be generated.

Advantages and Disadvantages of MPEG-1. The greatest disadvantage-which may be an advantage-is its resolution, limited to $\sim 320 \times 240$ pixels. It's a disadvantage because the resolution does not match typical dimensions of video (640X480 or $720 \mathrm{X} 486$ pixels, NTSC). But, the larger dimensions of video also require a more robust computer. The smaller dimensions of the MPEG-1 do not require that kind of power, and, in this sense, the MPEG-1 works to our advantage because we are assured smooth playback on ANY computer. Anyway, the image can be zoomed up two times without seeing much deterioration in the image quality.

Another advantage lies in its method for compressing. In my own experience, MPEG-1 compression retains detail without looking pixilated. Sorenson compression and Cinepak don't typically look as good. Much of that, however, depends on the nature of the images.

The use of MPEG-1 begs the question about MPEG-2 (the DVD format/CODEC). MPEG-2 has the advantage of greater resolution (720X576), and it would appear to be the best choice. While that would seem, by itself, to be most convincing, it only addresses resolution without considering other disadvantages. It does not speak at all to the quandaries surrounding the production of DVDs. If the DVD is only used to store the movie as a data file (and NOT a file that automatically plays a movie, such as what might occur were we to get a DVD-movie from a video store and put it in a DVD deck), then storing to a DVD disk is not a big deal. But don't expect the DVD itself to play on every computer with a DVD player. Because the technology is still growing, several details have not been worked out. For one, the recordable media itself is differentiated by DVD minus (-) R, DVD plus (+) R, and DVD plus or minus R. DVDs owe their fast growth to Macintosh and the Pioneer companies, and these DVDs use the minus $\mathrm{R}$ recordable media. Sony got into the act and decided upon its recordable media, plus $\mathrm{R}$. Then Sony made decks that playback both minus R and plus R DVDs. Therefore, older playback units are made only to play DVD-R, not DVD $+R$ (the only close-to-universal recordable disk is the DVD-R because it was the first on the market).

If the MPEG- 2 file is written to a DVD-R or $+\mathrm{R}$ using a program that includes code to tell the computer or DVD deck to auto-play a movie (such as Final Cut Pro), then matters get worse. These programs typically include so many dialogue boxes with so many choices; it takes a great deal of time and wasted DVDs to finally have success. The learning curve is so steep; many opt to send these out to local companies instead of dealing with the frustration of learning yet more minutia.

One final problem still lies at the door for those who want their videos on DVD, and that has to do with what was already mentioned: smooth playback. While that problem may not appear when replaying the movie for a colleague inside the comfort of an office, the playback with the additional detail of sending video signal to a laptop projector at a crowded meeting might result in jerky playback. One way to resolve that problem, or the problem of not seeing video at all, can be through changing the settings so that the laptop screen is turned off in favor of sending the video signal exclusively to the laptop projector. But that may not solve it, either.

Finally, the $320 \times 240$ MPEG-1 file can be zoomed to $200 \%$, making it $740 \times 480$ pixels. If the highest quality settings are used for compressing the MPEG-1 file, then the difference between MPEG-2 at $100 \%$ and MPEG-1 at $200 \%$ should be almost indistinguishable. Try it on your video to see for yourself.

The decision to have higher resolution, then, must be weighed against confidence in the computer's ability to playback the video smoothly. Anyway, in the end, movies do not need nearly the resolution demanded by still images. It's surprising how small video display

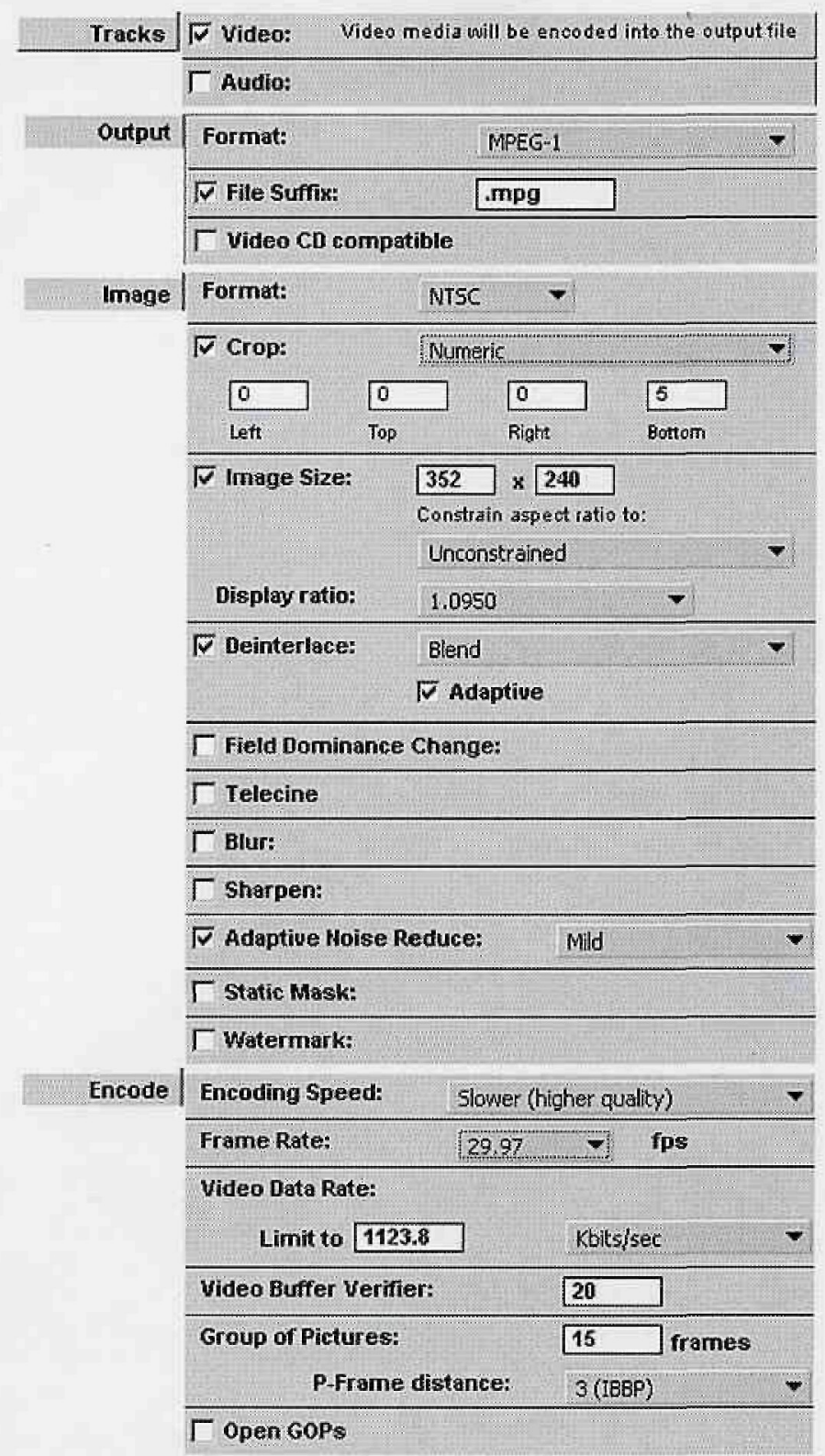

Caption, Figure 1. Possible options for MPEG-1 encoding. First select Track and uncheck Audio. Next choose the Image tab and choose the format (NTSC or PAL); choose Manual Crop and put in values to block video artifacts at the bottom and sides of the screen; choose Deinterlace and Adaptive if the movie was recorded from an analog, video signal. Choose the Slowest Speed/Highest Quality for encoding. These settings are part of the Media Cleaner Pro software program. 
sizes can be and we do not notice, for motion is much more easily detected by eye than small details in still images.

One other MPEG format has been the buzz lately, and that is the MPEG-4 format. Like any other newer introduction, considerations must be weighed in regard to replaying a format that needs a downloaded program. Furthermore, this format is generally regarded as inferior to REAL and Windows Media Video.

Converting Files to MPEG-1. Several programs can be used to convert from one video format to another. A simple search at download.com results in a list of programs starting at $\$ 25.00$. The industry standard is Media Cleaner Pro at $\sim \$ 500.00$, but Media Cleaner Light comes bundled with Adobe Premiere at a cheaper price, and it works for most applications.

These programs may be set up to give you several choices depending on the output of your movie; whether intended for the web or CD. These selections may make all the choices for you, but the results are often better if you make some choices yourself, when you are able. Here is a guide for making some of these selections in Media Cleaner (see also Figure 1):

Web or CD. What has not been mentioned thus far in regard to lowering the file size for smooth playback are two other parameters: the dimensions of the video display and the frame rate (frames per second). Display sizes and frame rates are lowest for the web and highest for DVD. If Web is chosen, and you are given a choice for the type of internet connection, it may be best to choose a rate that is lower than what is projected as the "typical" user. I choose phone line at $56 \mathrm{k}$.

Track: Be sure to unclick Audio, unless your movie includes audio.

Image: Size. The web image size will be quite small if you go with $56 \mathrm{k}$ for the internet connection. In any case, go with the suggested image dimensions. For a CD, the image will be at $320 \times 240$ (or thereabouts).

Crop. I choose Manual Crop and come up 5 pixels from the bottom (with $320 \times 240$ ), and sometimes from the sides. Artifacts appear at the edges of the screen (typically the bottom only) when the original source is videotape or digital video.

Deinterlace. Be sure to choose this, along with Adaptive, when the source is a video camera (as opposed to a video camera using digital media, such as mini-dv).

Encoding: Be certain to choose Slowest for the highest quality. The frame rate can be changed to make smaller file sizes, something to consider when making the movie available for download on the web, but not when the file will be written to CD.

While the movie is being encoded, it's best to let the computer clunk through the process without working on other programs. Otherwise, frames on the video might drop out.

GIF animation. Much has already been written about the creation of this kind of animation, and any search on the web will reveal a multitude of articles. A word of advice, however: most people over 25 do not care for continuous animation while attempting to read written material on a web page. A still photo of the first image in the GIF stack can be used as a link to another page in which the animation plays continuously. Be sure to include a Back button.

If the creation of MPEG-1 files becomes more trouble than it's worth, or if the results are not acceptable, then the MOV or AVI formats should also be tried, compressed or not. In most instances in which encoding is used, I have been far more satisfied with the MOV format, but this is a decision best made by each individual. However, in my own experience working in a core facility with researchers from varied disciplines, the MPEG-I has consistently been of the highest quality, least subject to jerky playback - the format I can confidently distribute.

\section{Expertise and joie de vivre... at} McGill University in Montréal, Canada

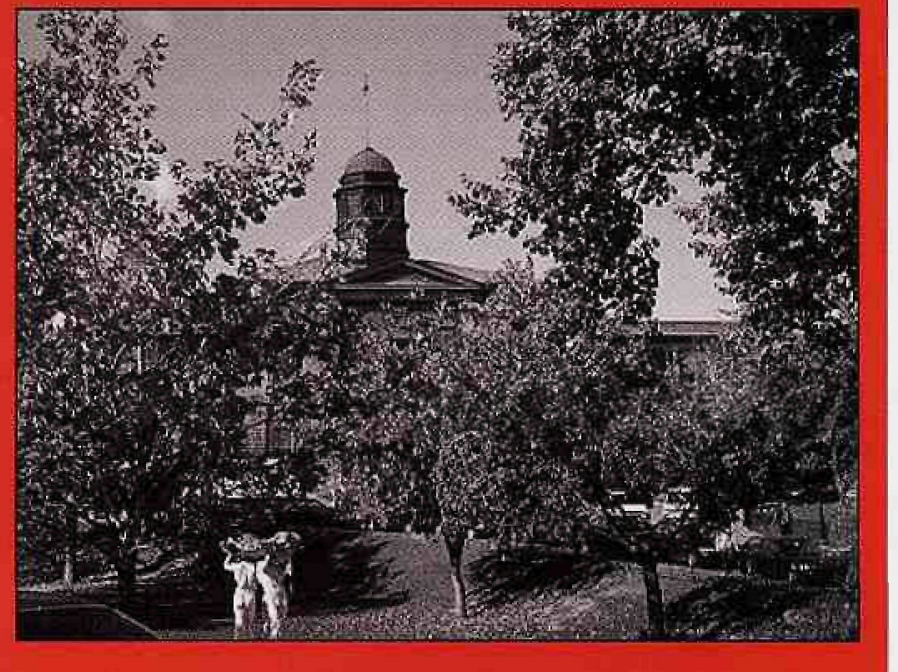

\section{"Advanced Techniques in SEM and Microanalysis for Materials Characterization"}

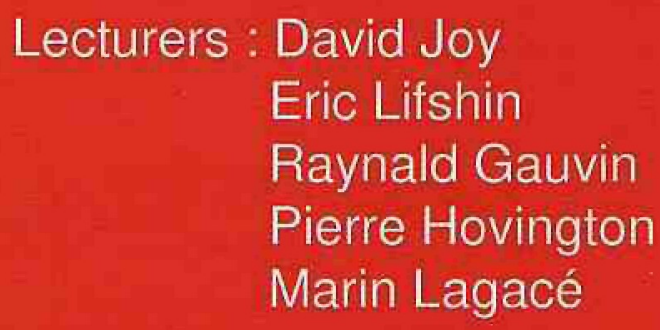

Registration deadline : 15 April 2004
Information : Prof. Raynald Gauvin

(514) $398-8951$

raynald.gauvin@megill.ca

http:/www. mogill.ca/minmet/ebeamworkshop 